\title{
Risankizumab in the treatment of psoriasis - literature review
}

\section{Katarzyna Banaszczyk}

Faculty of Medicine, Ludwik Rydygier Collegium Medicum in Bydgoszcz, Nicolaus Copernicus University in Torun, Bydgoszcz, Poland

\begin{abstract}
Risankizumab is a humanized, monoclonal antibody directed against subunit p19 of interleukin 23 (IL-23). In February 2019, risankizumab was approved for the treatment of moderate to severe psoriasis. The aim of the work is to collect up-to-date information on risankizumab and present its mechanism of action and recent clinical trials in which it was applied. This work also compares the mechanisms of action of risankizumab and ustekinumab and their importance in the treatment of psoriasis and describes the role of IL-23 in the etiopathogenesis of psoriasis. The work also refers to the effectiveness of risankizumab treatment and its safety profile. The results of molecular and histological studies that show changes in psoriatic skin after risankizumab treatment are also described.
\end{abstract}

Key words: psoriasis, antibodies, treatment, risankizumab.

\section{Introduction}

Psoriasis is a systemic, chronic inflammatory disease inseparably associated with the immune system, which is typically characterized by a cutaneous manifestation. At the present time, psoriasis is quite a challenge for modern medicine because it is often associated with a significant decrease in the quality of life and may even lead to disability [1]. In developed countries, the incidence of psoriasis is estimated at about 1-4\% [2]. It should be noted that psoriasis is associated with the risk of co-morbidities such as depression, heart disease, including ischemic heart disease, metabolic syndrome, hypertension and also inflammatory bowel diseases [3].

Psoriasis can co-exist with diseases such as psoriatic arthritis, inflammatory bowel diseases and ankylosing arthritis. Psoriatic arthritis affects $5 \%$ to $42 \%$ of patients with psoriasis. Interestingly, it can overtake skin symptoms in up to $15 \%$ of patients [4].

Due to the above reasons, intensive clinical trials are underway for new drugs, including antibodies, in order to best control the disease and increase the quality of life of patients with psoriasis. In recent years, the knowledge about the etiopathogenesis of psoriasis has significantly increased. The discovery of the prominent role of the interleukin (IL) 23/IL-17 immune axis in the pathogenesis of psoriasis has given a new option for the development of new therapies [5, 6].

The aim of this study is to gather current knowledge about the recently registered antibody risankizumab, which, as clinical trials show, seems to be a promising therapeutic option in the treatment of psoriasis.

\section{The mechanism of action of risankizumab and the role of IL-23 in the pathogenesis of psoriasis}

Risankizumab is a humanized, monoclonal IgG1 class antibody whose action is based on binding to the p19 subunit of IL-23. After binding to the antibody, IL-23 is selectively blocked, thus inhibiting its pro-inflammatory activity [7].

Interleukin 23 plays an important role in the development of chronic inflammation and, as genetic research has shown, there is a relationship between the receptor for IL-23 (IL-23R) or its ligand and inflammatory diseases, including psoriasis [8]. Interleukin 23 is involved in stimulation and has an effect on the functioning of Th17 lymphocytes, which play an important role in the pathogenesis of psoriasis [9].

Address for correspondence:

Katarzyna Banaszczyk, Faculty of Medicine, Ludwik Rydygier Collegium Medicum in Bydgoszcz, Nicolaus Copernicus University in Toruń, 13 Jagiellońska St., 85-067 Bydgoszcz, Poland, e-mail: kbanaszczyk570@gmail.com

Submitted: 3.04.2019; Accepted: 6.06.2019 
The development of inflammation and the immune response in psoriasis is associated with IL-23. According to the research, lack of IL-23 is associated with stopping the development of Th17 cells at the early stage of activation, which is associated, inter alia, with a reduction in the level of pro-inflammatory IL-17 produced by stimulated Th17 lymphocytes. The lack of IL-23 was also associated with a smaller pool of Th17 lymphocytes in the lymph nodes [10]. Interleukin 23 is necessary for the full inflammatory function of Th17 lymphocytes [11]. Moreover, it is worth noting that IL-23 is involved in the induction of IL-C3 cell activity, which also has significance in the pathogenesis of psoriasis [12]. The IL-23/IL-17 axis has been identified in the study as the major signaling pathway leading to characteristic molecular, cellular and structural changes in psoriatic skin [13]. Studies in mice showed that intradermal injection of IL-23 was associated with the development of psoriatic skin lesions [14].

Considering the play of cytokines and immune pathways, IL-23 seems to be a proper target for antibodies used to treat inflammatory diseases, including psoriasis.

\section{The mechanism of action of ustekinumab}

It is also worth mentioning the action of ustekinumab, which in clinical trials is compared with risankizumab in terms of safety and efficacy in the treatment of psoriasis. Ustekinumab is a fully human lgG1 monoclonal antibody that works by binding to the p40 subunit, common to IL-12 and IL-23 cytokines. It is worth noting that secondary inhibition of IL-12 may weaken the anti-inflammatory effect of this antibody. There are studies that indicate that IL-12 may in some way have anti-inflammatory action and inhibit dermatitis [15]. Interleukin 12 has a protective effect against intracellular pathogens, and is also likely to be involved in immune surveillance in the development of tumors $[16,17]$. Furthermore, inhibition of the p19 subunit is also associated with inhibition of IL-39, which is still a poorly studied factor [18].

Further research is needed to find out the properties and role of IL-39 in the pathogenesis of psoriasis, because it may turn out that this secondary action through the p19 subunit will render risankizumab superior to ustekinumab in the treatment of psoriasis. Bearing in mind the above reports, it may seem that the selective inhibition of IL-23 only is more beneficial and may be associated with fewer side effects during long-term treatment of psoriasis.

\section{Reports from the latest clinical trials}

In recent years, there have been several large clinical trials of risankizumab that have given hope of finding a new, effective drug for patients with psoriasis.
Krueger [19] and others focused on the safety assessment of risankizumab compared to placebo. The study included 39 patients aged 18-75 years with moderate to severe plaque psoriasis who had been suffering for at least 6 months. It was a one-stage, multi-center, double-blind, placebo-controlled clinical trial, in which patients received $0.01 \mathrm{mg} / \mathrm{kg}, 0.05 \mathrm{mg} / \mathrm{kg}, 0.25 \mathrm{mg} / \mathrm{kg}$, $1 \mathrm{mg} / \mathrm{kg}, 3 \mathrm{mg} / \mathrm{kg}$, or $5 \mathrm{mg} / \mathrm{kg}$ of risankizumab intravenously $(n=18), 0.25 \mathrm{mg} / \mathrm{kg}$ or $1 \mathrm{mg} / \mathrm{kg}$ risankizumab subcutaneously $(n=13)$, or matched placebo $(n=8)$. The effectiveness of treatment with this antibody was also evaluated. At week 12, Psoriasis Area Severity Index (PASI) 75 was achieved in $87 \%$ of cases, PASI 90 in 58\%, PASI 100 in $16 \%$. None of these end points were achieved in the placebo group. At week 24, PASI 75 was obtained in $71 \%$ of cases, PASI 90 in $48 \%$ and PASI 100 in 29\% of patients, compared to $13 \%$ for PASI 75 and 0\% for PASI 90 and 100 in the placebo group.

Regarding the safety profile, after 24 weeks, 20 out of 31 (65\%) patients receiving risankizumab (intravenously or subcutaneously) had an adverse event, while in the placebo group this percentage was $88 \%$ (7 out of 8 patients). The severity of adverse events did not correlate with the dose of risankizumab. The most frequent adverse events were upper respiratory tract infections, rhinitis and pharyngitis, i.e. adverse reactions that occur classically during the use of biological drugs in the course of psoriasis. Four serious events occurred during risankizumab use (alcoholic pancreatitis, ischemic stroke, transient ischemic attack and polymyositis), but they did not result from the treatment.

The above study showed that risankizumab was well tolerated and was associated with rapid and sustained clinical improvement in patients with moderate to severe plaque psoriasis. It is also worth noting that the use of risankizumab was associated with a decrease in the expression of genes and proteins associated with the IL-17/IL-23 axis [20]. This study included a relatively small group of patients, which is why in subsequent months the focus was on the next, larger clinical trials of risankizumab and its role in the treatment of psoriasis.

Another study, this time comparing risankizumab and ustekinumab, was carried out by Papp et al. [20]. It was a multicenter, randomized trial in which $166 \mathrm{pa}-$ tients with moderate or severe psoriasis participated. Patients were randomly assigned to the group with subcutaneous risankizumab (single dose $18 \mathrm{mg}$ or $90 \mathrm{mg}$ or $180 \mathrm{mg}$ at 0, 4 and 16 weeks) or ustekinumab (45 mg or $90 \mathrm{mg}$, depending on body weight, at week 0, 4, and 16). The primary endpoint for this study was the PASI 90 response or greater at week 12 of the study. At week 12, PASI 90 was achieved by $77 \%$ of patients (64 out of 84 ) using risankizumab at a dose of $90 \mathrm{mg}$ or $180 \mathrm{mg}$. In 
the ustekinumab group, this point was reached by $40 \%$ of patients (16 out of 40). PASI 100 was achieved in $45 \%$ of patients in the risankizumab $90 \mathrm{mg}$ or $180 \mathrm{mg}$ group, compared to $18 \%$ in the ustekinumab group. The effectiveness of treatment with risankizumab was maintained for approximately 20 weeks (the group receiving $90 \mathrm{mg} / 180 \mathrm{mg}$ ). It is also worth noting that patients us ing risankizumab reported a reduction in joint pain and nail condition improvement, which significantly affects the quality of life of patients with psoriasis [20]. This relatively short clinical trial has shown that risankizumab is more effective in the treatment of moderate to severe psoriasis compared to ustekinumab.

The next multicentre, double-blind, placebo-controlled trial compared the efficacy and safety of risankizumab in the treatment of moderate to severe plaque psoriasis. In this study, the researchers evaluated the efficacy of risankizumab compared to placebo and ustekinumab. The first phase of the study included 506 patients who were at least 18 years old and had moderate to severe plaque psoriasis. Patients were randomly assigned to receive subcutaneously either $150 \mathrm{mg}$ of risankizumab ( $n=304), 45 \mathrm{mg}$ or $90 \mathrm{mg}$ of ustekinumab $(n=100)$ or placebo $(n=102)$. In the second phase of the study, 491 patients were assigned to the group receiving subcutaneously either $150 \mathrm{mg}$ of risankizumab ( $n=$ 294), 45 mg or 90 mg of ustekinumab $(n=99)$ or placebo $(n=98)$. The end point was the proportion of patients achieving a 90\% improvement in PASI 90.

In the first phase of the study after 16 weeks in the risankizumab group 229 (75\%) of 304 patients achieved a $90 \%$ improvement in PASI. In the placebo group, the end-point was reached by 5 out of 100 patients, and in the group treated with ustekinumab 42 patients (42\%) out of 100 . In the second phase of the study, in the risankizumab 220 (75\%) group, 294 patients achieved the PASI 90 endpoint, compared with 47 (48\%) out of 98 in the ustekinumab group. Noteworthy is the fact that 109 (36\%) patients receiving risankizumab achieved complete regression of symptoms (PASI 100), while in the group using ustekinumab PASI 100 was achieved by 12 patients (12\%).

In the second phase of the study, total regression (PASI 100) was achieved in 149 (51\%) patients receiving risankizumab, compared to 24 patients (24\%) using ustekinumab. At week 52 of the study, in the first phase 249 (82\%) of patients receiving risankizumab achieved PASI 90, compared to 44 (44\%) receiving ustekinumab. Phase two after 52 weeks of treatment showed the following results - PASI 90 was obtained by 237 (81\%) patients using risankizumab and 50 patients (51\%) using ustekinumab. Complete regression of symptoms after 52 weeks (PASI 100) was obtained by 171 (56\%) patients in the first phase receiving risankizumab, compared to 21\% of patients treated with ustekinumab (second phase $60 \%$ respectively - risankizumab and 30\% - ustekinumab). The safety profile and the frequency of adverse events were similar for risankizumab, ustekinumab and placebo throughout the study and there were no unexpected safety findings [21]. This study showed that risankizumab was significantly more effective in treating plaque psoriasis compared to placebo and ustekinumab, without showing any serious side effects.

Visvanathan et al. [22] studied the molecular and histopathological profile of skin lesions in patients with psoriasis who had been treated with risankizumab and ustekinumab. Histological, immunohistochemical and genetic skin sections of 81 patients with moderate to severe psoriasis were analyzed. These patients were involved in two clinical trials with risankizumab and ustekinumab. Studies have shown that as early as the $4^{\text {th }}$ week of treatment with risankizumab (subcutaneously at a dose of 90 or $180 \mathrm{mg}$ ), histopathological expression of biomarkers such as K16, Ki67, CD3, lipocalin 2, CD11c, membrane glycoprotein associated with dendritic lysosome, p-defensin 2 and S100A7 was significantly lower. Significant improvement of these molecular parameters was noted in $54 \%$ of patients (risankizumab dose $90 \mathrm{mg}$ ) and in 69\% of patients (dose $180 \mathrm{mg}$ ).

In the ustekinumab group, this effect was achieved by $29 \%$ of patients. It was also noted that in the $4^{\text {th }}$ week of treatment risankizumab more strongly than ustekinumab decreased the expression of genes related to keratinocytes, epidermal cells and monocytes [22]. The study shows that risankizumab has stronger molecular activity than ustekinumab, which may indicate its higher effectiveness in the treatment of psoriasis.

\section{Risankizumab, Crohn's disease and psoriatic arthritis - reports from recent studies}

Recent clinical reports indicate that risankizumab may be an effective therapeutic option in the treatment of comorbidities with psoriasis, such as psoriatic arthritis and Crohn's disease.

Feagan et al. [23] conducted an interesting study on the efficacy of risankizumab in patients with moderately-to-severely active Crohn's disease. It was a randomized, double-blind, placebo-controlled study that included patients between 18 and 75 years of age, diagnosed with moderate-to-severe Crohn's disease lasting at least 3 months. Patients were divided into three groups in a $1: 1: 1$ ratio. Patients received intravenously risankizumab 200 mg, 600 mg or placebo. The intervention 
took place at week 0, 4 and 8 of the study. The primary endpoint was clinical remission $(\mathrm{CDAl}<150)$ at week 12 . At week 12 of the study, 25 out of 82 patients with risankizumab (total 41 patients in $200 \mathrm{mg}$ and 41 patients in the $600 \mathrm{mg}$ arms) had clinical remission compared to six (15\%, respectively) of the 39 patients in the placebo group. This short study shows that risankizumab was more effective than placebo in the induction of clinical remission in patients with active Crohn's disease [23].

In subsequent clinical trials of risankizumab and its effectiveness in the treatment of Crohn's disease, encouraging results were also obtained. 101 patients who completed the study described above were divided into three groups (33 patients in the placebo group, 34 in the $200 \mathrm{mg}$ risankizumab group and 34 in the $600 \mathrm{mg}$ risankizumab group). They received intravenous therapy with 600 mg risankizumab; the drug was administered every 4 weeks for 12 weeks. Remission induction was achieved in a larger proportion of patients at week 26 of the study compared to week 12: 18 (55\%) compared to $6(18 \%)$ of 33 patients in the original placebo group; $20(59 \%)$ vs. 7 (21\%) of 34 patients in the primary risankizumab group 200 mg; and 16 (47\%) vs. 9 (26\%) of 34 patients in the original $600 \mathrm{mg}$ risankizumab group. Of the 62 patients who received subcutaneous maintenance therapy (180 mg risankizumab), at week 52, 44 patients (71\%) maintained clinical remission, 50 (81\%) had a clinical response, 22 (35\%) had endoscopic remission, and 34 (55) \%) had an endoscopic response [24]. The study shows that risankizumab may be an effective therapy for patients with moderate to severe Crohn's disease, but more studies are needed to confirm its safety and efficacy in the treatment of this disease.

Promising results have also been obtained with respect to the treatment of psoriatic arthritis. In a randomized, double-blind, 24-week study, including patients with active psoriatic arthritis $(n=185)$, risankizumab $150 \mathrm{mg}$ administered subcutaneously significantly improved joint and skin symptoms compared to placebo. The primary endpoint was an improvement of $\geq 20 \%$ in the American College of Rheumatology (ACR20) criteria at week 16 of the study. An improvement of $\geq 20 \%$ at week 16 among risankizumab dosage groups (150 mg at weeks 0, 4, 8, 12, and 16; $150 \mathrm{mg}$ at weeks 0, 4, and 16; $150 \mathrm{mg}$ at weeks 0 and 12; and a $75 \mathrm{mg}$ single dose at week 0) was achieved in 57.1\%, 61.9\%, 59.0\% and $65.0 \%$ of patients, respectively, compared with $35.7 \%$ in the placebo group $[25,26]$. The above studies show that risankizumab is also effective in relieving and treating psoriasis-related diseases.

\section{Conclusions}

Clinical studies indicate that risankizumab may be a good therapeutic option in the treatment of moderate to severe plaque psoriasis. What is more, it is characterized by a very good safety profile and significant efficacy in the treatment of psoriasis, which exceeds the efficacy of ustekinumab. In February 2019 risankizumab after postive assessement by the Committee for Medicinal Products for Human Use (CHMP) was approved for the treatment of psoriasis by the Committee for Medicinal Products EMA, so it is worth considering a therapeutic option using this drug [27]. The above clinical trials proved that it is a very effective and safe therapeutic option.

The author declares no conflict of interest.

\section{References}

1. Boehncke W-H, Schön MP. Psoriasis. Lancet 2015; 386: 983994.

2. Parisi R, Symmons DP, Griffiths CE, et al. Global epidemiology of psoriasis: a systematic review of incidence and prevalence. J Invest Dermatol 2013; 133: 377-385.

3. Takeshita J, Grewal S, Langan SM, et al. Psoriasis and comorbid diseases: epidemiology. J Am Acad Dermatol 2017; 76: 377-390.

4. Habif TP. Psoriasis and other papulosquamous diseases. In: Clinical Dermatology, Habif TP (ed.), $6^{\text {th }}$ ed. Elsevier, Philadelphia, PA 2016: 263-328.

5. Blauvelt A, Chiricozzi A. The Immunologic Role of IL-17 in Psoriasis and Psoriatic Arthritis Pathogenesis. Clin Rev Allergy Immunol 2018; 55: 379-390.

6. Hawkes JE, Chan TC, Krueger JG. Psoriasis pathogenesis and the development of novel targeted immune therapies. J Allergy Clin Immunol 2017; 140: 645-653.

7. Singh S, Kroe-Barrett RR, Canada KA, et al. Selective targeting of the IL23 pathway: Generation and characterization of a novel high-affinity humanized anti-IL23A antibody. MAbs 2015; 7: 778-791.

8. Capon F, Di MP, Szaub J, et al. Sequence variants in the genes for the interleukin-23 receptor (IL23R) and its ligand (IL12B) confer protection against psoriasis. Hum Genet 2007; 122: 201-206.

9. Cargill M, Schrodi SJ, Chang M, et al. A large-scale genetic association study confirms IL12B and leads to the identification of IL23R as psoriasis-risk genes. Am J Hum Genet 2007; 80: 273-290.

10. McGeachy MJ, Chen Y, Tato CM, et al. The interleukin 23 receptor is essential for the terminal differentiation of interleukin 17-producing effector T helper cells in vivo. Nat Immunol 2009; 10: 314-324.

11. Ghoreschi K, Laurence A, Yang XP, et al. Generation of pathogenic $\mathrm{T}(\mathrm{H}) 17$ cells in the absence of TGF-beta signalling. Nature 2010; 467: 967-971. 
12. Teunissen MB, Munneke JM, Bernink JH, et al. Composition of innate lymphoid cell subsets in the human skin: enrichment of NCR(+) ILC3 in lesional skin and blood of psoriasis patients. J Invest Dermatol 2014; 134: 2351-2360.

13. Chan TC, Hawkes JE, Krueger JG. Interleukin 23 in the skin: role in psoriasis pathogenesis and selective interleukin 23 blockade as treatment. Ther Adv Chronic Dis 2018; 9: 111-119.

14. Chan JR, Blumenschein W, Murphy E, et al. IL-23 stimulates epidermal hyperplasia via TNF and IL-20R2-dependent mechanisms with implications for psoriasis pathogenesis. J Exp Med 2006; 203: 2577-2587.

15. Kulig P, Musiol S, Freiberger SN, et al. IL-12 protects from psoriasiform skin inflammation. Nat Commun 2016; 7: 13466.

16. Hamza T, Barnett JB, Li B. Interleukin 12 a key immunoregulatory cytokine in infection applications. Int J Mol Sci 2010; 11 789-806.

17. Lu X. Impact of IL-12 in Cancer. Curr Cancer Drug Targets 2017; 17: 682-697.

18. Wang X, Wei Y, Xiao H, et al. A novel IL-23p19/Ebi3 (IL-39) cytokine mediates inflammation in Lupus-like mice. Eur J Immunol 2016; 46: 1343-1350.

19. Krueger JG, Ferris LK, Menter A, et al. Anti-IL-23A mAb BI 655066 for treatment of moderate-to-severe psoriasis: safety, efficacy, pharmacokinetics, and biomarker results of a single-rising-dose, randomized, double-blind, placebo-controlled trial. J Allergy Clin Immunol 2015; 136: 116-124.

20. Papp KA, Blauvelt $A$, Bukhalo $M$, et al. Risankizumab versus ustekinumab for moderate-to-severe plaque psoriasis. N Engl J Med 2017; 376: 1551-1560.
21. Gordon KB, Strober B, Lebwohl M, et al. Efficacy and safety of risankizumab in moderate-to-severe plaque psoriasis (UltIMMa-1 and UltIMMa-2): results from two double-blind, randomised, placebo-controlled and ustekinumab - controlled phase 3 trials. Lancet 2018; 392: 650-661.

22. Visvanathan S, Baum P, Vinisko R, et al. Psoriatic skin molecular and histopathologic profiles after treatment with risankizumab versus ustekinumab. J Allergy Clin Immunol 2019; 143: 2158-2169.

23. Feagan BG, Sandborn WJ, D'Haens G, et al. Induction therapy with the selective interleukin-23 inhibitor risankizumab in patients with moderate-to-severe Crohn's disease: a randomised, double-blind, placebo-controlled phase 2 study. Lancet 2017; 389: 1699-1709.

24. Feagan BG, Panes J, Ferrantie M, et al. Risankizumab in patients with moderate to severe Crohn's disease: an open-label extension study. Lancet Gastroenterol Hepatol 2018; 3: 671-680.

25. Mease PJ, Kellner H, Morita A, et al. Efficacy and safety results from a phase 2 trial of risankizumab, a selective IL-23p19 inhibitor, in patients with active psoriatic arthritis. ACR/ARHP Annual Meeting 2017.

26. Mease P, Kellner H, Morita A, et al. OP0307 Efficacy and safety of risankizumab, a selective il-23p19 inhibitor, in patients with active psoriatic arthritis over 24 weeks: results from a phase 2 trial. Ann Rheum Dis 2018; 77: 200-201.

27. The European Medicines Agency. https://www.ema.europa. eu/en/medicines/human/summaries-opinion/skyrizi. 